\title{
Treatment of Generalized Pustular Psoriasis of Pregnancy With Infliximab
}

\author{
Burcu Beksac, MD, PhD; Esra Adisen, MD; Mehmet Ali Gurer, MD
}

\section{PRACTICE POINTS}

- Generalized pustular psoriasis of pregnancy (GPPP) is a rare and severe condition that may lead to complications in both the mother and the fetus. Effective treatment with low impact on the fetus is essential.

- Infliximab, among other biologic agents, may be considered for the rapid clearing of skin lesions in GPPP.

Generalized pustular psoriasis of pregnancy (GPPP) is a rare and severe condition that may impair the health of the mother and fetus. Effective treatment is essential, as treatment options for GPPP are limited due to concerns about unfavorable pregnancy outcomes. We report the case of a 22-year-old woman with GPPP that was unresponsive to systemic corticosteroids. We effectively treated the condition with infliximab at 30 weeks' gestation with an immediate clinical response and no detected serious adverse events except for an oral herpes infection in the patient and low birth weight in the neonate. Future studies are necessary to evaluate the safety and efficacy of infliximab treatment in GPPP.

Cutis. 2021;107:E2-E5.

$\longrightarrow$ eneralized pustular psoriasis of pregnancy (GPPP), formerly known as impetigo herpetiformis, is a rare dermatosis that causes maternal and fetal morbidity and mortality. It is characterized by widespread, circular, erythematous plaques with pustules at the periphery. ${ }^{1}$ Conventional first-line treatment includes systemic corticosteroids and cyclosporine. The National Psoriasis Foundation Medical Board also has included infliximab among the first-line treatment options for GPPP. ${ }^{2}$ Herein, we report a case of GPPP treated with infliximab at 30 weeks' gestation and during the postpartum period.

\section{Case Report}

A 22-year-old woman was admitted to our inpatient clinic at 20 weeks' gestation in her second pregnancy for evaluation of cutaneous eruptions covering the entire body. The lesions first appeared 3 to 4 days prior to her admission and dramatically progressed. She had a history of psoriasis vulgaris diagnosed during her first pregnancy 2 years prior that was treated with topical steroids throughout the pregnancy and methotrexate during lactation for a total of 11 months. She then was started on cyclosporine, which she used for 6 months due to ineffectiveness of the methotrexate, but she stopped treatment 4 months before the second pregnancy.

At the current presentation, physical examination revealed erythroderma and widespread pustules on the chest, abdomen, arms, and legs, including the intertriginous regions, that tended to coalesce and form lakes of pus over an erythematous base (Figure 1). The mucosae were normal. She exhibited a low blood pressure $(85 / 50 \mathrm{mmHg})$ and high body temperature $\left(102{ }^{\circ} \mathrm{F} \quad\left[38.9{ }^{\circ} \mathrm{C}\right]\right)$. Routine laboratory examination revealed anemia and a normal leukocyte count. Her erythrocyte sedimentation rate $(57 \mathrm{~mm} / \mathrm{h}$ [reference range, $<20 \mathrm{~mm} / \mathrm{h}$ ) ) and C-reactive protein level (102 mg/L

Dr. Beksac is from the Department of Dermatology, University of Health Sciences, Gulhane Training and Research Hospital, Ankara, Turkey. Dr. Adisen is from and Dr. Gurer was from the Department of Dermatology, Gazi University Faculty of Medicine, Ankara, Turkey. The authors report no conflict of interest.

Correspondence: Burcu Beksac, MD, PhD, Department of Dermatology, University of Health Sciences, Gulhane Training and Research Hospital, Gen. Dr. Tevfik Sanlam Caddesi, No:1, 06010 Kecioren/Ankara, Turkey (burcubeksac@gmail.com). 


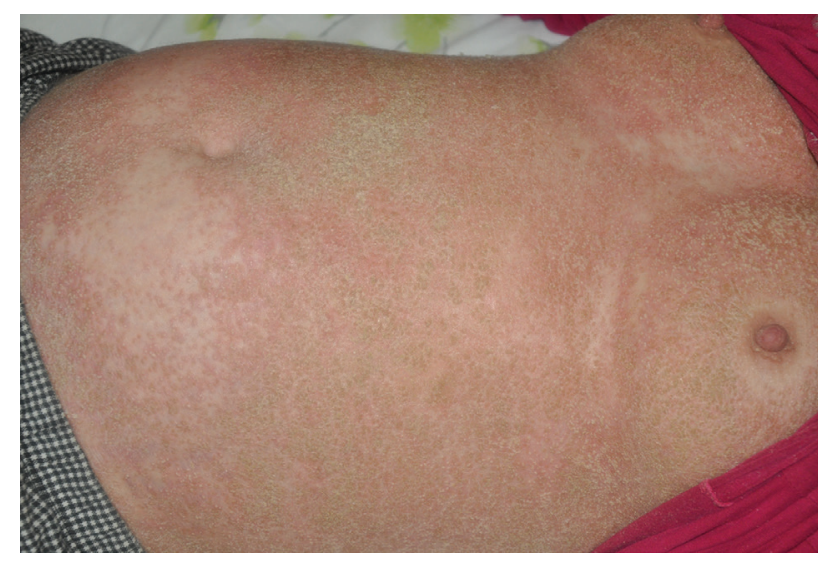

Generalized pustular psoriasis of pregnancy. Coalescing pustules and encrustations over an erythematous base on the abdomen.

[reference range, $<6 \mathrm{mg} / \mathrm{L}$ ]) were elevated, whereas total calcium $(8.11 \mathrm{mg} / \mathrm{dL}$ [reference range, $8.2-10.6 \mathrm{mg} / \mathrm{dL}$ ]) and albumin $(3.15 \mathrm{~g} / \mathrm{dL}$ [reference range, $>4.0 \mathrm{~g} / \mathrm{dL}$ ]) levels were low.

Empirical intravenous piperacillin/tazobactam was started due to hypotension, high fever, and elevated C-reactive protein levels; however, treatment was stopped after 4 days when microbiological cultures taken from blood and pustules revealed no bacterial growth, and therefore the fever was assumed to be caused by erythroderma. A skin biopsy before the start of topical and systemic treatment revealed changes consistent with GPPP.

Because her disease was extensive, systemic methylprednisolone $1.5 \mathrm{mg} / \mathrm{kg}$ once daily was started, and the dose was increased up to $2.5 \mathrm{mg} / \mathrm{kg}$ once daily on the tenth day of treatment to control new crops of eruptions. The dose was tapered to $2 \mathrm{mg} / \mathrm{kg}$ once daily when the lesions subsided 4 weeks into the treatment. The patient was discharged after 7 weeks at 27 weeks' gestation.

Twelve days later, the patient was readmitted to the clinic in an erythrodermic state. The lesions were not controlled with increased doses of systemic corticosteroids. Treatment with cyclosporine was considered, but the patient refused; thus, infliximab treatment was planned. Isoniazid $300 \mathrm{mg}$ once daily was started due to a risk of latent Mycobacterium tuberculosis infection revealed by a tuberculosis blood test. Other evaluations revealed no contraindications, and an infusion of infliximab $300 \mathrm{mg}(5 \mathrm{mg} / \mathrm{kg})$ was administered at 30 weeks' gestation. There was visible improvement in the erythroderma and pustular lesions within the same day of treatment, and the lesions were completely cleared within 2 days of the infusion. The methylprednisolone dose was reduced to $1.5 \mathrm{mg} / \mathrm{kg}$ once daily.

Three days after treatment with infliximab, lesions with yellow encrustation appeared in the perioral region and on the oral mucosa and left ear. She was diagnosed with an oral herpes infection. Oral valacyclovir $1 \mathrm{~g}$ twice daily and topical mupirocin were started and the lesions subsided within 1 week. Twelve days after the infliximab infusion, new pustular lesions appeared, and a second infusion of infliximab was administered 13 days after the first, which cleared all lesions within 48 hours.

The patient's methylprednisolone dose was tapered and stopped prior to delivery at 34 weeks' gestation2 weeks after the second dose of infliximab-as she did not have any new skin eruptions. A third infliximab infusion that normally would have occurred 4 weeks after the second treatment was postponed for a Cesarean section scheduled at 36 weeks' gestation due to suspected intrauterine growth retardation. The patient stayed at the hospital until delivery without any new skin lesions. The gross and histopathologic examination of the placenta was normal. The neonate weighed $4.8 \mathrm{lb}$ at birth and had neonatal jaundice that resolved spontaneously within 10 days but was otherwise healthy.

The patient returned to the clinic 3 weeks postpartum with a few pustules on erythematous plaques on the chest, abdomen, and back. At this time, she received a third infusion of infliximab 8 weeks after the second dose. For the past 5 years, the patient has been undergoing infliximab maintenance treatment, which she receives at the hospital every 8 weeks with excellent response. She has had no further pregnancies to date.

\section{Comment}

Generalized pustular psoriasis of pregnancy is a rare condition that typically occurs in the third trimester but also can start in the first and second trimesters. It may result in maternal and fetal morbidity by causing fluid and electrolyte imbalance and/or placental insufficiency, resulting in an increased risk for fetal abnormalities, stillbirth, and neonatal death. ${ }^{3}$ In subsequent pregnancies, GPPP has been observed to recur at an earlier gestational age with a more severe presentation. ${ }^{1,3}$

Generalized pustular psoriasis of pregnancy usually involves an eruption that begins symmetrically in the intertriginous areas and spreads to the rest of the body. The lesions present as erythematous annular plaques with pustules on the periphery and desquamation in the center due to older pustules. ${ }^{1,3}$ The mucous membranes also may be involved with erosive and exfoliative plaques, and there may be nail involvement. Patients often present with systemic symptoms such as fever, malaise, diarrhea, and vomiting. ${ }^{1}$ Laboratory investigations may reveal neutrophilic leukocytosis, high erythrocyte sedimentation rate, hypocalcemia, and hypoalbuminemia. ${ }^{4}$ Cultures from blood and pustules show no bacterial growth. A skin biopsy is helpful in diagnosis, with features similar to generalized pustular psoriasis, demonstrating spongiform pustules containing neutrophils, lymphocytic and neutrophilic infiltrates in the papillary dermis, and negative direct immunofluorescence. ${ }^{3}$

The differential diagnosis of GPPP includes subcorneal pustular dermatosis, dermatitis herpetiformis, herpes gestationis, impetigo, and acute generalized exanthematous 
pustulosis. ${ }^{1,3}$ Due to concerns of fetal implications, treatment options in GPPP are somewhat limited; however, the condition requires treatment because it may result in unfavorable pregnancy outcomes. Topical corticosteroids may be an option for limited disease.,6 Systemic corticosteroids (eg, prednisone $60-80 \mathrm{mg} / \mathrm{d}$ ) were previously considered as first-line agents, although they have shown limited efficacy in our case as well as in other case reports. ${ }^{7}$ Their ineffectiveness and risk for flare-up after dose tapering should be kept in mind when starting GPPP patients on systemic corticosteroids. Systemic cyclosporine $(2-3 \mathrm{mg} / \mathrm{kg} / \mathrm{d})$ may be added to increase the efficacy of systemic steroids, which was done in several cases in literature. ${ }^{1,6,8}$ Although cyclosporine has been classified as a pregnancy category C drug, an analysis of pregnancy outcomes of 629 renal transplant patients revealed no association with adverse pregnancy outcomes compared to the general population and no increase in fetal malformations. ${ }^{9}$ Therefore, cyclosporine is a safe treatment option and was classified as a first-line drug for GPPP in a 2012 review by the National Psoriasis Foundation Medical Board. ${ }^{2}$ Narrowband UVB also has been reported to be used for the treatment of GPPP. ${ }^{10}$ Methotrexate and retinoids have been used in cases with lesions that persisted postpartum. ${ }^{1}$

Anti-tumor necrosis factor (TNF) $\alpha$ agents are another effective option for treatment of GPPP. Anti-TNF agents are classified as pregnancy category $\mathrm{B}$ due to results showing that anti-mouse TNF- $\alpha$ monoclonal antibodies did not cause embryotoxicity or teratogenicity in pregnant mice. ${ }^{11}$ Although Carter et a ${ }^{12}$ published a review of US Food and Drug Administration data on pregnant women receiving anti-TNF treatment and concluded that these agents were associated with the VACTERL group of malformations (vertebral defects, anal atresia, cardiac defect, tracheoesophageal fistula with esophageal atresia, cardiac defects, renal and limb anomalies), no such association was found in further studies. A 2014 study showed no difference in the rate of major malformations in infants born to women who were treated with anti-TNF drugs compared to the disease-matched group not treated with these agents and pregnant women counselled for nonteratogenic exposure. ${ }^{13}$ The same study detected an increase in preterm and low-birth-weight deliveries and suggested this might be caused by the increased severity of disease in patients requiring anti-TNF medication. The British Society of Rheumatology Biologics Register published data on pregnancy outcomes in 130 rheumatoid arthritis patients who had been exposed to anti-TNF agents. ${ }^{14}$ The results suggested an increased rate of spontaneous abortions in women exposed to anti-TNF treatment around the time of conception, especially in those taking these medications together with methotrexate or leflunomide; however, results also indicated that disease activity may have had an impact on the rate of spontaneous abortions in these patients. In a 2013 review of 462 women with inflammatory bowel disease who had been exposed to anti-TNF agents during pregnancy, the investigators concluded that pregnancy outcomes and the rate of congenital anomalies did not significantly differ from other inflammatory bowel disease patients not receiving anti-TNF drugs or the general population. ${ }^{15}$

In 2012, the National Board of the National Psoriasis Foundation put infliximab amongst the first-line treatment modalities for GPPP. ${ }^{2}$ In one case of GPPP in which the eruption persisted after delivery, the patient was treated with infliximab 7 weeks postpartum due to failure to control the disease with prednisolone $60 \mathrm{mg}$ daily and cyclosporine $7.5 \mathrm{mg} / \mathrm{kg}$ daily. Unlike our patient, this patient was only started on an infliximab regimen after delivery.$^{16}$ In another case reported in 2010, the patient was started on infliximab during the postpartum period of her first pregnancy following a pustular flare of previously diagnosed plaque psoriasis (not a generalized pustular psoriasis, as in our case). ${ }^{17}$ As a good response was obtained, infliximab treatment was continued in the patient throughout her second pregnancy.

Our case is unique in that infliximab was started during pregnancy because of intractable disease leading to systemic symptoms. Our patient showed an excellent response to infliximab after a 10 -week disease course with repeated flare-ups and impairment to her overall condition. Delivery occurred at 36 weeks' gestation due to suspected intrauterine growth retardation; however, the neonate was born with a 5-minute APGAR score of 10 and required no special medical care, which suggests that the low birth weight was constitutional due to the patient's small frame (her height was $4 \mathrm{ft} 11 \mathrm{in}$ ). The breast milk of patients with inflammatory bowel disease has been detected to contain very small amounts of infliximab $(101 \mathrm{ng} / \mathrm{mL}$, about 1/200 of the therapeutic blood level) ${ }^{18}$ Considering the large molecular weight of this agent and possible proteolysis in the stomach and intestines, infliximab is unlikely to affect the neonate. ${ }^{15}$ Thus, we encouraged our patient to breastfeed her baby. A case of fatal disseminated Bacille-Calmette-Guérin infection in an infant whose mother received infliximab treatment during pregnancy has been reported. ${ }^{19}$ It has been suggested that live vaccines should be avoided in neonates exposed to anti-TNF agents at least for the first 6 months of life or until the agent is no longer detectable in their blood. ${ }^{15}$ We therefore informed our patient's family practitioner about this data.

\section{Conclusion}

We report a case of infliximab treatment for GPPP that was continued during the postpartum period. Infliximab was an effective treatment option in our patient with no detected serious adverse events and may be considered in other cases of GPPP that are not responsive to systemic steroids. However, further studies are warranted to evaluate the safety and efficacy of infliximab treatment for GPPP and psoriasis in pregnancy. 


\section{REFERENCES}

1. Lerhoff $\mathrm{S}$, Pomeranz MK. Specific dermatoses of pregnancy and their treatment. Dermatol Ther. 2013;26:274-284.

2. Robinson A, Van Voorhees AS, Hsu S, et al. Treatment of pustular psoriasis: from the Medical Board of the National Psoriasis Foundation. J Am Acad Dermatol. 2012;67:279-288.

3. Oumeish OY, Parish JL. Impetigo herpetiformis. Clin Dermatol. 2006;24:101-104.

4. Gao QQ, Xi MR, Yao Q. Impetigo herpetiformis during pregnancy: a case report and literature review. Dermatology. 2013;226:35-40.

5. Bae YS, Van Voorhees AS, Hsu S, et al. Review of treatment options for psoriasis in pregnant or lactating women: from the Medical Board of the National Psoriasis Foundation. J Am Acad Dermatol. 2012;67:459-477.

6. Shaw CJ, Wu P, Sriemevan A. First trimester impetigo herpetiformis in multiparous female successfully treated with oral cyclosporine [published May 12, 2011]. BMJ Case Rep. doi:10.1136/bcr.02.2011.3915

7. Hazarika D. Generalized pustular psoriasis of pregnancy successfully treated with cyclosporine. Indian J Dermatol Venereol Leprol. 2009;75:638.

8. Luan L, Han S, Zhang Z, et al. Personal treatment experience for severe generalized pustular psoriasis of pregnancy: two case reports. Dermatol Ther. 2014;27:174-177.

9. Lamarque V, Leleu MF, Monka C, et al. Analysis of 629 pregnancy outcomes in transplant recipients treated with Sandimmun. Transplant Proc. 1997;29:2480

10. Bozdag K, Ozturk S, Ermete M. A case of recurrent impetigo herpetiformis treated with systemic corticosteroids and narrowband UVB. Cutan Ocul Toxicol. 2012;31:67-69.
11. Treacy G. Using an analogous monoclonal antibody to evaluate the reproductive and chronic toxicity potential for a humanized anti-TNF alpha monoclonal antibody. Hum Exp Toxicol. 2000;19:226-228.

12. Carter JD, Ladhani A, Ricca LR, et al. A safety assessment of tumor necrosis factor antagonists during pregnancy: a review of the Food and Drug Administration database. J Rheumatol. 2009;36:635-641.

13. Diav-Citrin O, Otcheretianski-Volodarsky A, Shechtman S, et al. Pregnancy outcome following gestational exposure to TNF-alpha-inhibitors: a prospective, comparative, observational study. Reprod Toxicol. 2014;43:78-84.

14. Verstappen SM, King Y, Watson KD, et al. Anti-TNF therapies and pregnancy: outcome of 130 pregnancies in the British Society for Rheumatology Biologics Register. Ann Rheum Dis. 2011;70:823-826.

15. Gisbert JP, Chaparro M. Safety of anti-TNF agents during pregnancy and breastfeeding in women with inflammatory bowel disease. Am J Gastroenterol. 2013;108:1426-1438.

16. Sheth N, Greenblatt DT, Acland K, et al. Generalized pustular psoriasis of pregnancy treated with infliximab. Clin Exp Dermatol. 2009;34:521-522.

17. Puig L, Barco D, Alomar A. Treatment of psoriasis with anti-TNF drugs during pregnancy: case report and review of the literature. Dermatology. 2010;220:71-76.

18. Ben-Horin S, Yavzori-M, Kopylov U, et al. Detection of infliximab in breast milk of nursing mothers with inflammatory bowel disease. J Crohns Colitis. 2011;5:555-558.

19. Cheent K, Nolan J, Shariq S, et al. Case report: fatal case of disseminated BCG infection in an infant born to a mother taking infliximab for Crohn's disease.J Crohns Colitis. 2010;4:603-605. 